\title{
Variation in rotavirus vaccine coverage by sub-counties in Kenya
}

\author{
Ernest Apondi Wandera ${ }^{1,2^{*}}$, Shah Mohammad ${ }^{1}$, John Odhiambo Ouko ${ }^{1}$, James Yatitch ${ }^{3}$, Koki Taniguchi ${ }^{4}$ \\ and Yoshio Ichinose ${ }^{1}$
}

\begin{abstract}
Rotavirus gastroenteritis is an important cause of childhood morbidity and mortality in Kenya. In July 2014, Kenya introduced the rotavirus vaccine into her national immunization program. Although immunization coverage is crucial in assessing the real-world impact of this vaccine, variability in the vaccine coverage across the country is likely to occur. In view of this, we estimated the extent of coverage for the rotavirus vaccine at two socio-economically different sub-counties using the administrative data. The findings indicate disparities in vaccine coverage and access between the sub-counties and, thus, underscore the need to strengthen immunization systems to facilitate timely, accessible, and equitable vaccine delivery across the country. Both sub-counties recorded high vaccine dropout, suggestive of poor utilization of the vaccine. In this regard, increased social mobilization is needed to encourage vaccine compliance and to enhance tracking of vaccine defaulters. While efforts to improve the accuracy of the administrative coverage estimates are crucial, vaccination coverage surveys will be needed to verify the administrative coverage data and help identify specific factors relating to rotavirus vaccine coverage in the country.
\end{abstract}

Keywords: Kenya, Rotavirus vaccine, Administrative data, Coverage, Dropout

\section{Background}

The rotavirus is the most common cause of severe diarrhea among children $<5$ years globally and is estimated to cause 215,000 deaths annually [1]. In Kenya, the rotavirus is estimated to cause more than 3908 deaths [1], 3015 outpatient visits, and 279 hospitalizations per 100,000 children $<5$ years [2] and to cost the healthcare system US\$ 10.8 million annually [3]. Safe and effective rotavirus vaccines are considered to be important tools for reducing the burden of diarrhea and, thereby, contributing to the achievement of the Sustainable Development Goal 3 [4, 5]. In July 2014, Kenya introduced Rotarix $^{\circ}$, a two-dose rotavirus vaccine into her Expanded Program on Immunization (EPI). The vaccine is administered orally at 6 and 10 weeks of age [6]. Over the first 20 years of its introduction in Kenya, the rotavirus vaccine is predicted to avert 60,935 undiscounted deaths and 216,454 hospital admissions among children aged

\footnotetext{
* Correspondence: wandesh2000@yahoo.com

${ }^{1}$ KEMRI/Nagasaki University, Institute of Tropical Medicine, Kenya Research

Station, P.O. Box 19993-00202, Nairobi, Kenya

${ }^{2}$ Leading Program, Graduate School of Biomedical Sciences, Nagasaki

University, Nagasaki, Japan

Full list of author information is available at the end of the article
}

$<5$ years and help the government avoid a healthcare cost of US\$ 30 million and a cost per disability-adjusted life year (DALY) of US\$ 38 million [7].

Nevertheless, a high vaccination coverage is essential to maximize the impact of the rotavirus vaccine. In countries with high levels of immunization coverage, the impact of rotavirus vaccines in reducing the burden of severe childhood diarrhea has been remarkable [8-10]. Conversely, in low-income settings like Kenya, vaccine coverage rates are likely to be lower due to programmatic, geographical, and social challenges [11]. According to the WHO/UNICEF [12], the rotavirus vaccine achieved only $38 \%$ coverage in $50 \%$ of the national target Kenyan population in 2014 and $66 \%$ in 2015 . There was a countrywide dropout rate of $5.8 \%$ with eight counties reporting $>10 \%$ dropout. However, given the fact that Kenya is a socio-economically diverse country [13], variability in rotavirus vaccine coverage across the subcounties would not be surprising. In view of this, we estimated rotavirus vaccine coverage at Kiambu, a periurban sub-county, and Mbita, a rural sub-county. 


\section{Main text}

\section{Methods}

Kiambu sub-county is located in Kiambu County, Central Kenya, on the outskirts of Nairobi, the capital of Kenya. According to the World Bank ranking, Kiambu is the second richest county in Kenya, with an estimated GDP of 3.0 billion US dollars (USD), which accounts for $11 \%$ of the national GDP [13]. The major economic activities in the sub-county are agriculture and industries. According to the 2014 Kenya Demographic and Health Survey (KDHS) [14], 82.8\% of children aged 12-23 months in this area were fully vaccinated. Mbita sub-county is located on the shores of Lake Victoria in Homa Bay County, Western Kenya, and about $400 \mathrm{~km}$ west of Nairobi. Homa Bay County is among the poorest in Kenya with its GDP of 0.3 billion USD accounting for only $1.2 \%$ of the national GDP [13]. Less than $25 \%$ of the adult population has a regular wage employment. The population depends on subsistence farming, small-scale businesses, fishing, and keeping domestic animals [15]. According to the 2014 KDHS, only $53.7 \%$ of children aged $12-23$ months in this area were fully immunized [14].

We obtained the administrative data on rotavirus vaccination from the Units of Vaccines and Immunization Services (UVISs) for Kiambu and Mbita sub-counties. The administrative data included the target population aged $<1$ year which is eligible for rotavirus vaccination, the monthly targets for rotavirus vaccination, and the monthly doses (1 and 2$)$ of rotavirus vaccine routinely administered to the target population in each of the sub-counties as summarized in Table 1. Using these data, we estimated the percentage of rotavirus immunization coverage and dropout rates in Kiambu and Mbita sub-counties between August 2014 and April 2016. Coverage, which is the proportion of vaccinated individuals among the target population, was calculated by dividing the number of individuals vaccinated with rotavirus vaccine dose 1 or 2 (numerator) by the number of individuals targeted for vaccination (denominator) within the same period (month/year). This proportion was then multiplied by 100 to obtain the percentage coverage. Dropout rate, which refers to the number of individuals who start an immunization schedule but fail to get the last dose on the schedule, was calculated by subtracting the number of individuals who received the last dose of rotavirus vaccine from the number of individuals who received the first dose of the vaccine (numerator) and dividing the difference by the number of individuals who received the first dose of the vaccine (denominator). This proportion was then multiplied by 100 to obtain the percentage dropout [16]. Access to rotavirus vaccination (the children who are reached by immunization services) was measured using percentage coverage and described as either good $(\geq 80 \%)$ or poor $(<80 \%)$. Utilization of rotavirus vaccine (the ability to retain the children accessed by the vaccine until they receive the last dose on the schedule) was measured using the dropout rate and described as either high (when the dropout rate was $<10 \%$ ) or low (when the dropout rate was $\geq 10 \%$ ) [16].

\section{Results}

There was a distinct variation in the extent of rotavirus vaccine coverage between Kiambu and Mbita subcounties. In Kiambu sub-county, the vaccine coverage for 2014 was $107.4 \%$ for dose 1 and $82.4 \%$ for dose 2, representing a dropout rate of $23.3 \%$. In 2015 , the coverage for doses 1 and 2 was 127.2 and $116.3 \%$, respectively, with a dropout rate of $8.6 \%$. In 2016, the coverage was 136.7 and $124.3 \%$ for doses 1 and 2, respectively, with a dropout of $9.1 \%$ (Table 1). Overall, the coverage for doses 1 and 2 in Kiambu sub-county was 124.4 and $109.8 \%$, respectively, with a dropout rate of $11.7 \%$

Table 1 Rotavirus vaccine targets, doses administered, and coverage in Kiambu and Mbita sub-counties, August 2014-April 2016

\begin{tabular}{|c|c|c|c|c|c|c|c|c|}
\hline Sub-county & Year & Monthly target ${ }^{a}$ & Yearly target ${ }^{b}$ & No. of dose $1^{c}$ & $\%$ coverage for dose 1 & No. of dose $2^{d}$ & $\%$ coverage for dose 2 & Dropout (\%) \\
\hline \multirow[t]{4}{*}{ Kiambu } & 2014 & 285 & 1425 & 1530 & 107.4 & 1174 & 82.4 & 23.3 \\
\hline & 2015 & 285 & 3420 & 4351 & 127.2 & 3977 & 116.3 & 8.6 \\
\hline & 2016 & 295 & 1180 & 1613 & 136.7 & 1467 & 124.3 & 9.1 \\
\hline & Total & & 6025 & 7494 & 124.4 & 6618 & 109.8 & 11.7 \\
\hline \multirow[t]{4}{*}{ Mbita } & 2014 & 402 & 2010 & 1096 & 54.5 & 667 & 33.2 & 39.1 \\
\hline & 2015 & 414 & 4968 & 3821 & 76.9 & 3348 & 67.4 & 12.4 \\
\hline & 2016 & 426 & 1704 & 1327 & 77.9 & 1156 & 67.8 & 12.9 \\
\hline & Total & & 8682 & 6244 & 71.9 & 5171 & 59.6 & 17.1 \\
\hline
\end{tabular}

${ }^{a}$ Number of children aged $<1$ year being targeted for rotavirus vaccination in a month in each sub-county

${ }^{b}$ Number of children aged $<1$ year being targeted for rotavirus vaccination in a year in each sub-county. 2014, 2015, and 2016 totals include 5 months (August-December)

12 months (January-December), and 4 months (January-April), respectively, and form the denominator for calculating the vaccine coverage

c,d Number of individuals vaccinated with rotavirus doses 1 and 2 in a year in each sub-county. 2014, 2015, and 2016 totals include 5 months (August-December),

12 months (January-December), and 4 months (January-April), respectively, and form the numerator for calculating the vaccine coverage

${ }^{a, b}, c, d$ Compiled from administrative data obtained from the Unit of Vaccines and Immunization Services (UVIS), Kiambu and Mbita sub-counties 
(Table 1$)$. Thus, the high coverage $(\geq 80 \%)$ demonstrates good access to the rotavirus vaccine in this sub-county whereas the high dropout rate $(\geq 10 \%)$ indicates a slightly poor utilization of the vaccine in this area.

In Mbita sub-county, rotavirus vaccination coverage for 2014 was $54.5 \%$ for dose 1 and $33.2 \%$ for dose 2, representing a dropout rate of $39.1 \%$. In 2015, the coverage increased for both doses 1 and 2 at 76.9 and $67.4 \%$, respectively, with a dropout rate of $12.4 \%$. In 2016, the coverage increased to 77.9 and $67.8 \%$ for doses 1 and 2, respectively, with a dropout of $12.9 \%$ (Table 1 ). On average, the coverage in Mbita sub-county for doses 1 and 2 was 71.9 and $59.6 \%$, respectively, with a dropout rate of 17.1\% (Table 1). Thus, Mbita sub-county recorded a slightly poor access and utilization of the rotavirus vaccine. Both sub-counties recorded a steady increase in monthly rotavirus vaccination coverage for both dose 1 and dose 2 over the study period (Fig. 1).

\section{Discussion}

In this report, we demonstrate varied rotavirus vaccine coverage between Kiambu and Mbita sub-counties in Kenya which are socio-economically different. Kiambu sub-county recorded higher rotavirus vaccination coverage than Mbita sub-county. Disparities in coverage for other childhood vaccines have been reported from various Demographic and Health Surveys (DHSs) in Kenya and have been attributed mainly to the socioeconomic status of the child's family and maternal occupation and education [14, 17-19]. In a recent nationwide DHS, full immunization coverage, especially for the series vaccines, was highest among children from households in the highest wealth quintile (71\%) [14]. Kiambu sub-county is wealthier than Mbita sub-county [13-15]. Furthermore, the countrywide survey reported higher immunization coverage in Kiambu than Mbita for both the first and the second doses of the pentavalent, polio, and pneumococcal vaccines [14] that are given alongside the rotavirus vaccine. These factors might explain the higher coverage of rotavirus vaccine in Kiambu than Mbita. The high coverage in Kiambu sub-county indicates good access to the vaccine and might be attributed to enhanced social mobilization within the community. The sub-county UVIS organizes monthly community outreach programs aimed at sensitizing the community on the importance of vaccinations among other health matters, thereby generating demand for vaccines and improving the visibility of immunization among the community. In addition, several community health officers $(\mathrm{CHOs})$ and community health volunteers $(\mathrm{CHVs})$ are engaged in conducting health talks in the community and tracing vaccine defaulters, which have also helped lower the dropout rate.

On the other hand, Mbita sub-county recorded a low rotavirus vaccine coverage, suggesting a poor access to the vaccine. The low coverage might be attributed to the delayed introduction of the rotavirus vaccine, adherence to age restrictions, shortage of health workers, frequent human migrations, and limited cold-chain capacity at some facilities in the sub-county [20]. However, the coverage improved with subsequent years partly due to an enhanced social mobilization and door-to-door health promotion activities by the sub-county's UVIS. The vaccine dropout was more pronounced for Mbita subcounty, indicating poor utilization of the rotavirus vaccine in this area. Various DHSs in Kenya have

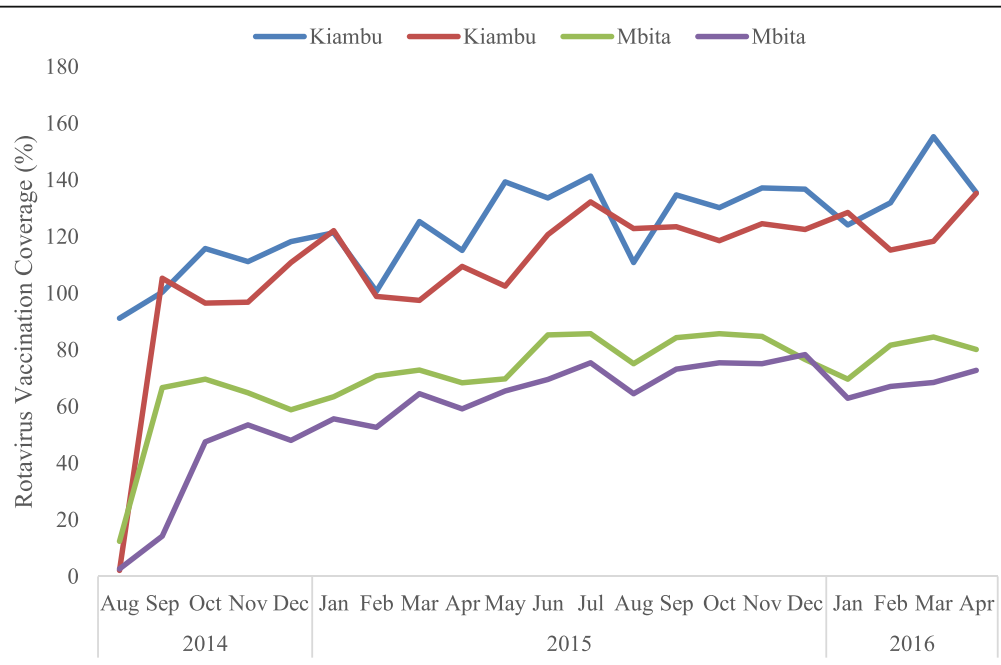

Fig. 1 Trends in monthly rotavirus vaccination coverage in Kiambu and Mbita sub-counties, 2014-2016. The graph compares the trends in monthly rotavirus immunization coverage rates between Kiambu and Mbita sub-counties as estimated from the vaccine administrative data for doses 1 and 2 between August 2014 and April 2016. The vaccine administrative data was used with permission from the Kiambu and Mbita sub-counties' Units of Vaccines and Immunization Services (UVIS) 
observed that the coverage for series vaccinations declined with subsequent doses [14, 17, 19]. The high vaccine dropout in Mbita sub-county might be partly attributed to the constant mobility of the fishing community in the sub-county. Thus, the sub-county $\mathrm{CHOs}$ and CHVs should liaise with the fishing community leaders in the public health education programs and in the follow-up of vaccine defaulters.

In this report, vaccine administrative data obtained at the sub-county level were used to estimate the rotavirus vaccine coverage in Kiambu and Mbita. However, there are limitations to the accuracy of immunization coverage estimates derived from the administrative data [21-24]. Hence, the coverage recorded in this report for the rotavirus vaccine could be higher than the actual coverage due to a possible overestimation of the numerators and/ or underestimation of the denominators. Overestimation of the numerators might occur due to over-reporting from the peripheral health facilities. In Kenya, the accuracy of the proportion of immunizations reported at the central (sub-county/national) level that can be tracked down to the peripheral health facilities, expressed as the verification factor (VF), has been found to be $85 \%$ (95\% CI, 68-103\%) [23]. We could not verify the coverage data from the peripheral health facilities that were reported to the sub-counties' UVIS. Overestimation of the numerators could also occur as a result of vaccinating children outside the target age group or target catchment population. Underestimation of the denominators could result from inaccurate vaccine target setting due to population movements and/or inaccurate census projections-Kenya conducts a national census only after 10 years. Thus, the accuracy of the administrative coverage estimates should be improved through regular data quality assessments to avoid errors in recording, compiling, and reporting vaccinations at the peripheral health facilities to higher levels and accurate vaccine target setting by accurate projections of the catchment population. In addition, vaccination coverage surveys should be conducted to verify the administrative coverage estimates [21-24].

\section{Conclusions}

There was a disparity in rotavirus vaccine coverage between the two socio-economically distinct subcounties; hence, efforts should be made to strengthen the immunization system to facilitate equitable delivery of vaccination services across the country. The high vaccine dropout in both sub-counties underscores the need for enhanced community outreach programs that will encourage those who receive the first dose of the vaccine to return for the remaining dose and facilitate effective tracing of any vaccine defaulters.

\section{Abbreviations}

CHOs: Community health officers; CHVs: Community health volunteers; DALY: Disability-adjusted life year; DHS: Demographic and Health Survey; EPI: Expanded Program on Immunization; GDP: Gross domestic product; KDHS: Kenya Demographic and Health Survey; KEMRI: Kenya Medical Research Institute; UNICEF: United Nations Children's Fund; USD: United States dollar; UVIS: Unit of Vaccines and Immunization Services;

VF: Verification factor; WHO: World Health Organization

\section{Acknowledgements}

We are grateful to the Director of the Kenya Medical Research Institute (KEMRI) for the collaborative support. We wish to thank the Heads of the Unit of Vaccines and Immunization Services (UVIS) at Kiambu and Mbita subcounties in Kenya for their collaborative support.

\section{Funding}

This study was supported in part by Grant-in-Aid for Scientific Research, Japan (Research B: 15H05286) and the Program for Nurturing Global Leaders in TECD, Nagasaki University, Japan.

\section{Availability of data and materials}

The datasets used and/or analyzed during the current study are available from the corresponding author on reasonable request.

\section{Authors' contributions}

EAW and $\mathrm{Yl}$ conceptualized and designed the study and drafted the manuscript. $\mathrm{JO}$ and $\mathrm{JY}$ collected and analyzed the administrative data on rotavirus vaccination at the sub-counties. SM and KT edited and critically revised the manuscript. All authors read and approved the final version of the manuscript.

\section{Competing interests}

The authors declare that they have no competing interests.

\section{Consent for publication}

Not applicable.

Ethics approval and consent to participate

Not applicable.

\section{Publisher's Note}

Springer Nature remains neutral with regard to jurisdictional claims in published maps and institutional affiliations.

\section{Author details}

${ }^{1}$ KEMRI/Nagasaki University, Institute of Tropical Medicine, Kenya Research Station, P.O. Box 19993-00202, Nairobi, Kenya. 'Leading Program, Graduate School of Biomedical Sciences, Nagasaki University, Nagasaki, Japan. ${ }^{3}$ Public Health Department, Kiambu sub-county, Kiambu, Kenya. ${ }^{4}$ Department of Virology and Parasitology, School of Medicine, Fujita Health University, Toyoake, Japan.

Received: 17 November 2016 Accepted: 17 April 2017

Published online: 24 April 2017

References

1. Tate JE, Burton AH, Boschi-Pinto C, Steele AD, Duque J, Parashar UD, WHOcoordinated Global Rotavirus Surveillance Network. Global, regional, and national estimates of rotavirus mortality in children $<5$ years of age, 2000-2013. CID. 2016; 62 Suppl 2:96-105.

2. van Hoek AJ, Ngama M, Ismail A, Chuma J, Cheburet S, Mutonga D, Kamau T, Nokes DJ. A cost effectiveness and capacity analysis for the introduction of universal rotavirus vaccination in Kenya: comparison between Rotarix and RotaTeq vaccines. PLoS One. 2012;7:e47511. doi:10. 1371/journal.pone.0047511.

3. Tate JE, Rheingans RD, O'Reilly CE, Obonyo B, Burton DC, Tornheim JA, Adazu K, Jaron P, Ochieng B, Kerin T, Calhoun L, Hamel M, Laserson K, Breiman RF, Feikin DR, Mintz ED, Widdowson MA. Rotavirus disease burden and impact and cost-effectiveness of a rotavirus vaccination program in Kenya. J Infect Dis. 2009;200(Suppl):76-84.

4. Tessa W, Peter S, Clarissa B, Mickey C, Elizabeth M. Diarrhoea: why children are still dying and what can be done. Lancet. 2010;375:870-2. 
5. Rodrigo C, Salman N, Tatochenko V, Meszner Z, Giaquinto C. Recommendations for rotavirus vaccination: a worldwide perspective. Vaccine. 2010;28:5100-8.

6. GlaxoSmithKline. Summary of product characteristics. www.medicines.org. uk/EMC/medicine/17840/SPC/Rotarix/ (Accessed 11 August 2016).

7. Sigei C, Odaga J, Mvundura M, Madrid Y, Clark AD. Cost-effectiveness of rotavirus vaccination in Kenya and Uganda. Vaccine. 2015;Suppl 1:A109-18.

8. Patel MM, Glass R, Desai R, Tate JE, Parashar UD. Fulfilling the promise of rotavirus vaccines: how far have we come since licensure? Lancet Infect Dis. 2012;12:561-70.

9. Patel MM, Steele D, Gentsch JR, Wecker J, Glass RI, Parashar UD. Real-world impact of rotavirus vaccination. Pediatr Infect Dis J. 2011;30 Suppl 1:1-5.

10. Parashar UD, Johnson H, Steele AD, Tate JE. Health impact of rotavirus vaccination in developing countries: progress and way forward. CID. 2016;62 Suppl 2:91-5.

11. Cherian T, Wang S, Mantel C. Rotavirus vaccines in developing countries: the potential impact, implementation challenges, and remaining questions. Vaccine. 2012;30 Suppl 1:A3-6.

12. The WHO and UNICEF. 2015 Estimates of national immunization coverage: Kenya. www.who.int/immunization/monitoring_surveillance/data/ken.pdf (Accessed 19 August 2016)

13. Bundervoet T, Maiyo $L$ and Sanghi A. Bright lights, big cities: measuring national and subnational economic growth in Africa from outer space, with an application to Kenya and Rwanda. The World Bank, Kenya and Rwanda Country Management Unit, Macroeconomics and Fiscal Management Global Practice Group, and Poverty Global Practice Group. 2015. http://econ worldbank.org. (Accessed 7 April 2017).

14. Kenya National Bureau of Statistics (KNBS); ICF Macro, 2015. Kenya demographic and health survey 2014. Key Indicators 2014. https:// dhsprogram.com/pubs/pdf/FR308/FR308.pdf (Accessed 20 August 2016).

15. Wanyua S, Ndemwa M, Kensuke G, Junichi T, K'Opiyo J, Okumu S, Diela P, Satoshi K, Mohamed K, Ichinose Y, Shimada M. Profile: the Mbita health and demographic surveillance system. Int J Epidemiol. 2013;42:1678-85.

16. Republic of Kenya, Ministry of Health. Immunization manual for health workers. http://www.mchip.net/sites/default/files/mchipfiles/ Immunization\%20Manual\%20for\%20Health\%20Workers_updated.pdf (Accessed 21 Apr 2017).

17. Calhoun LM, van Eijk AM, Lindblade KA, Odhiambo FO, Wilson ML, Winterbauer E, Slutsker L, Hamel MJ. Determinants and coverage of vaccination in children in Western Kenya from a 2003 cross-sectional survey Am J Trop Med Hyg. 2014;90(2):234-41.

18. Mutua MK, Kimani-Murage E, Ettarh RR. Childhood vaccination in informal urban settlements in Nairobi, Kenya: who gets vaccinated? BMC Public Health. 2011:11:6-11.

19. Maina LC, Karanja S, Kombich J. Immunization coverage and its determinants among children aged 12-23 months in a peri-urban area of Kenya. Pan Afr Med J. 2013;14:3. doi:10.11604/pamj.2013.14.3.2181.

20. GAVI-Kenya Joint Appraisal Report, 2015. www.gavi.org/country/kenya/ documents/jas/joint-appraisal-kenya-2015/ (Accessed 1 September 2016).

21. Burton A, Monasch R, Lautenbach B, Gacic-Dobo M, Neill M, Karimov R, Wolfson L, Jones G, Birmingham M. WHO and UNICEF estimates of national infant immunization coverage: methods and processes. Bull World Health Organ. 2009;87:535-41.

22. Murray CIL, Shengelia B, Gupta N, Moussavi S, Tandon A, Thieren M. Validity of reported vaccination coverage in 45 countries. Lancet. 2003;362:1022-7.

23. Bosch-Capblanch X, Ronveaux O, Doyle V, Remedios V, Bchir A. Accuracy and quality of immunization information systems in forty-one low-income countries. Trop Med Int Health. 2009;14:2-10.

24. Cutts FT, Izurieta H, Rhoda DA. Measuring coverage in MNCH: design, implementation and interpretation challenges associated with tracking vaccination coverage using household surveys. PLoS Med. 2013:10(5): e1001404.

\section{Submit your next manuscript to BioMed Central and we will help you at every step:}

- We accept pre-submission inquiries

- Our selector tool helps you to find the most relevant journal

- We provide round the clock customer support

- Convenient online submission

- Thorough peer review

- Inclusion in PubMed and all major indexing services

- Maximum visibility for your research

Submit your manuscript at www.biomedcentral.com/submit

) Biomed Central 